\title{
All-semiconductor metamaterial with negative refraction in the near-infrared
}

\author{
Naik, Gururaj V.; Liu, Jingjing; Kildishev, Alexander V.; Shalaev, Vladimir M.; Boltasseva, Alexandra
}

Published in:

CLEO Technical Digest

Publication date:

2012

Document Version

Publisher's PDF, also known as Version of record

Link back to DTU Orbit

Citation (APA):

Naik, G. V., Liu, J., Kildishev, A. V., Shalaev, V. M., \& Boltasseva, A. (2012). All-semiconductor metamaterial with negative refraction in the near-infrared. In CLEO Technical Digest (pp. QTh1A.1). Optical Society of America.

\section{General rights}

Copyright and moral rights for the publications made accessible in the public portal are retained by the authors and/or other copyright owners and it is a condition of accessing publications that users recognise and abide by the legal requirements associated with these rights.

- Users may download and print one copy of any publication from the public portal for the purpose of private study or research.

- You may not further distribute the material or use it for any profit-making activity or commercial gain

- You may freely distribute the URL identifying the publication in the public portal 


\title{
All-semiconductor metamaterial with negative refraction in the near-infrared
}

\author{
Gururaj V. Naik ${ }^{1}$, Jingjing Liu ${ }^{1}$, Alexander V. Kildishev ${ }^{1}$, Vladimir M. Shalaev ${ }^{1}$ and Alexandra \\ Boltasseva $^{*, 1,2}$ \\ ${ }^{I}$ School of Electrical and Computer Engineering and Birck Nanotechnology Center, Purdue University, West Lafayette, IN 47907, USA \\ ${ }^{2}$ DTU Fotonik, Department of Photonics Engineering, Technical University of Denmark, Lyngby, DK-2800, Denmark \\ ${ }^{3}$ Erlangen Graduate School of Advanced Optical Technologies (SAOT), Universität Erlangen-Nürnberg, Paul-Gordan-Str. 6, 91052 Erlangen, \\ Germany \\ Email: $\underline{\text { aeb @ purdue.edu }}{ }^{*}$
}

\begin{abstract}
When heavily doped, semiconductors such as $\mathrm{ZnO}$ can exhibit metallic properties thus becoming versatile building blocks for optical metamaterials. Here, we design and fabricate an allsemiconductor metamaterial and demonstrate negative refraction in the near-infrared region. OCIS codes: (250.5403) Plasmonics; (160.3918) Metamaterials; (310.7005) Transparent Conductive Coatings;
\end{abstract}

\section{Introduction}

Plasmonics and metamaterials have brought a paradigm shift in the scope and outlook of optics by enabling novel functionalities such as sub-diffraction imaging, cloaking and optical magnetism [1]. However, these novel devices suffer from limitations arising from the optical properties of their metallic (plasmonic) components. Semiconductors can be good alternatives to traditional plasmonic materials if they can be tuned to exhibit metallic properties. Since semiconductors allow the effective control of their properties (e.g. by doping), recently there has been a rising interest in using them as the building blocks of plasmonic and metamaterial devices [2]. Advances in semiconductor technology have driven the rise of the information age with numerous solid-state devices such as the ubiquitous transistors and lasers used in everyday life. Now, all-semiconductor metamaterials could overcome the drawbacks of metal-dielectric composites and enable high-performance devices in the optical frequencies [2].

In general, semiconductors are dielectric at optical frequencies due to their relatively small number of charge carriers. However, they can exhibit metallic properties if sufficiently doped [3]. Achieving metallic properties in semiconductors in the near-infrared and visible wavelength ranges requires ultra-high doping $\left(10^{21} \mathrm{~cm}^{-3}\right.$ or higher). This is extremely difficult due to problems such as dopant solid-solubility limit. Also, higher doping is often associated with higher scattering loss, which is undesirable. This fact has hampered the transition of semiconductors into the realm of plasmonics and optical metamaterials. However, semiconductors such as zinc oxide (ZnO) can make the jump from electronics to plasmonics [2]. In this work, we have used aluminum-doped $\mathrm{ZnO}$ (AZO) as a low-loss plasmonic material and $\mathrm{ZnO}$ as a dielectric material to demonstrate negative refraction in an important class of metamaterials exhibiting hyperbolic dispersion (HMMs) [4]. The AZO/ZnO HMM is fabricated using pulsed laser deposition of sixteen 62-nm-thick alternating layers of $\mathrm{ZnO}$ and AZO (2 wt\%) on a silicon substrate. In the effective-medium limit, such a planar, lamellar structure exhibits uniaxial anisotropy. The directions parallel to the layers are characterized by a real effective permittivity $\left(\varepsilon_{\|}\right)$, which has a sign opposite to that of the perpendicular direction $(\varepsilon \perp)$, hence leading to hyperbolic dispersion. Figure $1 \mathrm{~A}$ shows the effective permittivity values of the $\mathrm{AZO} / \mathrm{ZnO}$ layered stack retrieved from spectroscopic ellipsometry (V-VASE, J. A. Woollam Co.). In the wavelength range from 1.8 to $2.4 \mu \mathrm{m}$, this layered stack exhibits hyperbolic dispersion $\left(\varepsilon^{\prime} \|>0, \varepsilon^{\prime} \perp<0\right.$, which is known as transverse negative HMM) under TM-polarized incident light. Transverse negative dispersion, although very challenging to realize with metal-dielectric composites, is essential for negative refraction [5-6] and highperformance imaging applications [7]. Our all-semiconductor transverse negative HMM is a viable alternative both for the visible and near-infrared wavelength ranges.

Figure $1 \mathrm{C}$ depicts the experimental set-up for observing negative refraction in the AZO/ZnO HMM. As a blade partially blocks the transmitted beam, the relative transmittance (ratio of transmittance with and without the blade) should dip if negative refraction were to occur. Figure 1B shows a pseudo-color map of measured relative transmittance for different incident angles and wavelength (TM). The contours show the matching profile from theoretical calculations. The dip in relative transmittance for the wavelength range from 1.8 to $2.4 \mu \mathrm{m}$ confirms negative refraction. No similar dip in relative transmittance is observed for any incident angle with TE-polarization. 
The performance of HMMs can be characterized by a figure-of-merit: FoM $=\operatorname{Re}\{\beta \perp\} / \operatorname{Im}\{\beta \perp\}$, where $\beta \perp$ is the propagation vector in the direction perpendicular to the layers [5]. The FoM for this AZO/ZnO HMM is about 12, which is at least three orders of magnitude larger than for HMMs designed using either silver or gold. In summary, we have designed and fabricated an all-semiconductor metamaterial, and we successfully demonstrated its high performance through a negative refraction experiment in the near-infrared wavelength region.
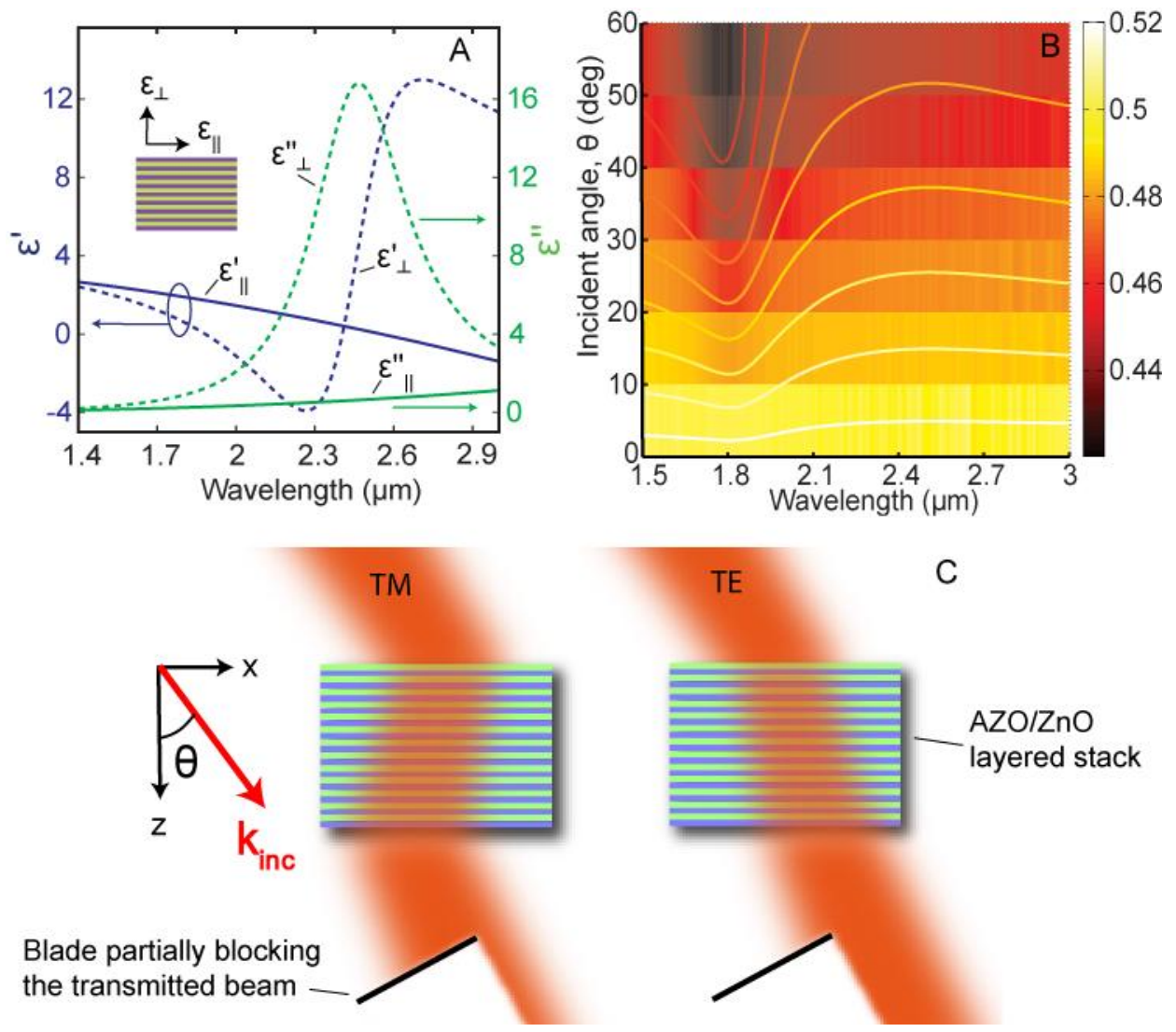

Fig 1. Characteristics of a planar hyperbolic metamaterial (HMM) formed by 16 alternating layers of $\mathrm{ZnO}$ and $\mathrm{Al}: \mathrm{ZnO}(\mathrm{AZO})$ of $62 \mathrm{~nm}$ each. (A) Extracted effective permittivities $\left(\varepsilon^{\prime}+\mathbf{i} \varepsilon^{\prime \prime}\right)$ in the parallel $(\|)$ and perpendicular $(\perp)$ directions. (b) Measured relative transmittance (ratio of transmittance with and without blade blocking half of the beam) in TM polarization for angles of incidence from $0,10,20,30,40,50$ and $60^{\circ}$. The contours show the theoretical predictions based on negative refraction in the HMM. (c) Experimental set-up for observing negative refraction. In TM polarization, the layered stack exhibits hyperbolic dispersion, which results in negative refraction. In TE polarization the dispersion is not hyperbolic, and normal refraction occurs.

Acknowledgements: This work was supported in part by ONR MURI grant N00014-10-1-0942 and ARO grant W911NF-11-1-0359.

\section{References}

[1] C. M. Soukoulis, et al., "Negative refractive index at optical wavelengths," Science, vol. 315, p. 47, 2007.

[2] A. Boltasseva and H. A. Atwater, "Low-loss plasmonic metamaterials," Science, vol. 331, p. 290, 2011.

[3] G. Naik and A. Boltasseva, "Semiconductors for plasmonics and metamaterials," physica status solidi (RRL)-Rapid Research Letters, vol. 4 , pp. 295-297, 2010.

[4] Z. Jacob, et al., "Optical Hyperlens: Far-field imaging beyond the diffraction limit," Opt. Express, vol. 14, pp. 8247-8256, 2006.

[5] A. Hoffman, et al., "Negative refraction in semiconductor metamaterials," Nature Materials, vol. 6, pp. 946-950, 2007.

[6] J. Yao, et al., "Optical negative refraction in bulk metamaterials of nanowires," Science, vol. 321, p. 930, 2008.

[7] Z. Liu, et al., "Far-field optical hyperlens magnifying sub-diffraction-limited objects," Science, vol. 315, p. 1686, 2007. 